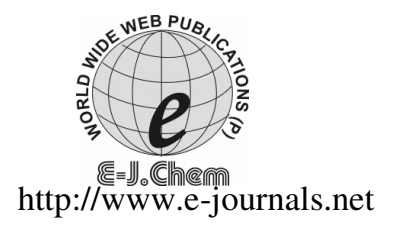

ISSN: 0973-4945; CODEN ECJHAO

E-Journal of Chemistry

2011, 8(4), 1574-1581

\title{
3-(Substituted Aryl)-1-benzofuranyl-2-propenones: Antimicrobial Properties of Some Chalcones-Type Compounds and their 2-Pyrazoline Derivatives
}

\author{
DEMET COŞKUN ${ }^{\S *}$, MISIR AHMEDZADE ${ }^{\S}$ and SEVDA KIRBAĞ \\ ${ }^{\S}$ University of Firat, Faculty of Science and Arts

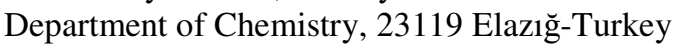 \\ University of Firat, Faculty of Science and Arts \\ Department of Biology, 23119 Elazığ-Turkey \\ dcoskun@firat.edu.tr
}

Received 13 November 2010; Accepted 19 January 2011

\begin{abstract}
Acetylbenzofuran on condensation with furan-2-carboxaldehyde and pyrrole-2-carboxaldehyde in methanolic $\mathrm{KOH}$ solution yielded the corresponding benzofuran chalcones. These two compounds and nine benzofuran chalcones were synthesized before, were further reacted with hydrazine hydrate in ethanol which led to the formation of 2-pyrazoline derivatives. All the synthesized compounds were characterized by elemental analysis, melting point determination, infrared spectroscopy and nuclear magnetic resonance spectroscopy. Nine chalcone-type compounds and eleven 2-pyrazolines were evaluated for their biological activities against the six bacteria and the three yeast and it was seen that thirteen compounds showed activity. Four of them are chalcone-type compounds showed more or less activity
\end{abstract}

Keywords: Benzofuran, Chalcone, Pyrazoline, Antimicrobial, Antifungal

\section{Introduction}

The various activities of the compounds possessing benzofuran, chalcone and pyrazoline moiety are well known. The pyrazoline function is quite stable and has inspired chemists to utilize this stable fragment in bioactive moieties to synthesize new compounds possessing biological activities. Chalcones have various biological activities such as antioxidant ${ }^{1,2}$, cytotoxic $^{3}$, antiviral ${ }^{4,5}$, tyrosinase inhibitory ${ }^{6}$, antimalarial ${ }^{7,8}$, antibacterial ${ }^{9,10}$ and antiinflammatory ${ }^{11,12}$. Pyrazolines have been also reported to show a broad spectrum of biological activities including antibacterial ${ }^{13,14}$, antifungal ${ }^{15}$, antioxidant ${ }^{14}$, anti-inflammatory ${ }^{15,16}$, cytotoxic $^{17}$, antidepressant ${ }^{18}$, anti-tumoral ${ }^{19}$ and hypotensive ${ }^{20}$ activities. 
There are lots of environmental bacteria capable of causing a variety of life threatening human infections. In this study, synthesis and characterization of two newly synthesized chalcone-type compouns, 1-(benzofuran-2-yl)-3-(furan-2-yl)-2-propenone (1j) and 1-(benzofuran2-yl)-3-(pyrrol-2-yl)-2-propenone (1k) are described. Preparation of 2-pyrazolines derivatives (2a-k) of these two compounds and the previously synthesized nine chalconetype compounds $\mathrm{s}^{21}$ are reported. These chalcones and their 2-pyrazoline derivatives were screened for their biological activities against six bacteria and three yeast.

\section{Experimental}

All chemicals were purchased from Fluka and Aldrich. All melting points were measured using a differential scanning calorimeter (Shimadzu DSC-50) and are uncorrected. Elemental analyses were performed on a Leco CHNS-932 apparatus. ${ }^{1} \mathrm{H}-$ and ${ }^{13} \mathrm{C}$ NMR spectra were recorded on a Bruker AC $300(300 \mathrm{MHz})$ and a Varian Mercury-Plas NMR $(400 \mathrm{MHz})$ in the specified deuterated solvents. Chemical shifts were expressed in ppm from internal tetramethysilane. Infrared (IR) spectra were recorded as $\mathrm{KBr}$ pellets on a PerkinElmer Spectrum One FTIR spectrometer.

General method for the synthesis of 3-(substituted aryl and heteroaryl)-1benzofuranyl-2-propenones $(\mathbf{1 j}$ and $1 \mathrm{k}$ )

To a mixture of 2-acetylbenzofuran $(2.187 \mathrm{~g}, 13.6 \mathrm{mmol})$ and the appropriate aldehyde $(13.6 \mathrm{mmol})$ in methanol $(25 \mathrm{~mL})$ a solution of sodium hydroxide $(1 \mathrm{M}, 18 \mathrm{~mL})$ was added. The reaction mixture was stirred for $3 \mathrm{~h}$ on a magnetic stirrer at room temperature and was allowed to stand in refrigerator for overnight. The product was precipitated by pouring to $250 \mathrm{~mL}$ of cold water, was filtered and dried and crystallized from ethanol.

General method for the synthesis of 3-(2-benzofuranyl)-5-aryl and heteroaryl-2pyrazolines $(\mathbf{2} \boldsymbol{a}-\boldsymbol{k})$

The pyrazolines were synthesized as follow; Compounds 2a-k: The required chalcone-type compound (example, 1a, $1.320 \mathrm{~g}, 5 \mathrm{mmol})$ was suspended in absolute ethanol $(10 \mathrm{~mL})$ and stirred at room temperature. hydrazine hydrate $(0.375 \mathrm{~g}, 7.5 \mathrm{mmol})$ was added and the mixture stirred at reflux for $30 \mathrm{~min}$. Upon cooling, the mixture was poured to cold water $(500 \mathrm{~mL})$ and the precipitate was filtered off and washed with water and dried. The product (2a) was crystallized from ethanol solution, yield: $1.029 \mathrm{~g}, 74 \%, \mathrm{mp}: 21{ }^{\circ} \mathrm{C}$.

The reflux time in the transformation of the chalcones to the pyrazolines varied from 10 to $60 \mathrm{~min}$ according to the kinds of the chalcones ( $10 \mathrm{~min}$ for $\mathbf{2 \mathbf { j }}$ and $\mathbf{2 e}, 15 \mathrm{~min}$ for $\mathbf{2 k}$, $30 \mathrm{~min} \mathbf{2 c}$ and $\mathbf{2 i}, 40 \mathrm{~min}$ for $\mathbf{2 a}, \mathbf{2 b}, \mathbf{2 d}, \mathbf{2 f}$ and $\mathbf{2} \mathbf{h}$ and $60 \mathrm{~min}$ for $\mathbf{2 g}$ ).

\section{Evaluation of biological action}

Six bacteria and three yeast were used as test organisms. These bacteria were Staphylococcus aureus COWAN 1, Pseudomonas aeruginosa DMS 50071 SCOTTA, Proteus vulgaris FMC 1, Escherichia coli ATCC 25922, Klebsiella pneumoniae FMC, Bacillus megaterium DMS 32, and the yeasts were Candida albicans FMC 17, Candida glabrata ATCC 66032 and Candida tropicalis ATCC 13803.

All of the synthesized compounds were screened against six bacteria and three yeasts using the method given in literature ${ }^{22}$. The bacteria strains were inoculated into nutrient broth and yeast strain inoculated into malt extract broth for 24 and $48 \mathrm{~h}$, respectively. In the disc- diffusion method, sterile Mueller-Hinton agar for bacteria and Malt extract agar for yeasts 
were separately inoculated with the test bacteria $\left(10^{5}\right.$ bacteria per $\left.\mathrm{mL}\right)$ and yeasts $\left(10^{4}\right.$ yeast per $\left.\mathrm{mL}\right)$. In order to prepare the disc a part containing $1000 \mu \mathrm{g}$ of compound from its solution in DMSO was added onto the discs. In addition, disk containing DMSO were placed $(10 \mu \mathrm{L})$. Discs were applied on the solid agar medium by pressing slightly. Petri dishes were placed at $4{ }^{\circ} \mathrm{C}$ for $2 \mathrm{~h}$. The bacteria samples were incubated at $35 \pm 0.1{ }^{\circ} \mathrm{C}$ for $24 \mathrm{~h}$ and yeast samples were incubated at $25 \pm 0.1^{\circ} \mathrm{C}$ for $72 \mathrm{~h}$. At the end of the period, inhibition zones were measured in millimeter. The inhibition zone of compound was calculated by subtracting that of pure DMSO from the zone of solution in DMSO of the compound.

\section{Results and Discussion}

This work is the second part of the studies on 3-(substituted aryl)-1-benzofuranl-2propenones and describes the synthesis and characterization of 2-pyrazoline derivatives (2a-k) from the chalcones (1a-i) given previously ${ }^{21}$ and two new chalcones (1j and $\left.\mathbf{1 k}\right)$ (Scheme 1) and the biological activities of the twenty products. The synthesized chalcones compounds were suspended in absolute ethanol, hydrazine hydrate was added and the mixture stirred at reflux for a period of 10-60 min as depending on the kind of chalcones. The cyclization of chalcones were carried out at a short period of time ${ }^{13,14}$. The cyclization of some chalcones with hydrazine hydrate was required to make a reflux of $7 \mathrm{~h}$ in ethanol ${ }^{13}$. 2-Pyrazoline derivatives were formed in 74-90\% yields ${ }^{13}$. The yields in formation of 3-(benzofuran-2yl)-5-(3-nitrophenyl)-2-pyrazoline (2f) and bispyrazoline (2h) are $60 \%$ and $45 \%$, respectively and relatively low.

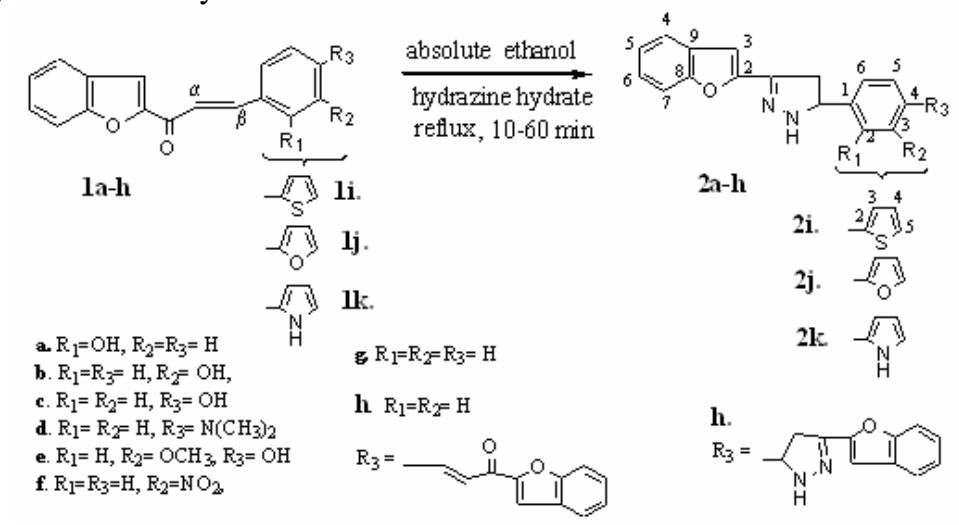

Scheme 1. 2-Pyrazoline derivatives of some chalcone-type compounds Structural characterization (FTIR, ${ }^{1} \mathrm{H}$ - and ${ }^{13} \mathrm{C}$-NMR)

The newly synthesized two benzofuran chalcones $(\mathbf{1 j}$ and $\mathbf{1 k})$ showed the characteristic absorption bands at $1659 \mathrm{~cm}^{-1}$ ( $\mathrm{C}=\mathrm{O}$ stretching in $\left.\mathbf{1 j}\right)$, at $1648 \mathrm{~cm}^{-1}(\mathrm{C}=\mathrm{O}$ stretching in $\mathbf{1 k})$, at $3000-3150 \mathrm{~cm}^{-1}$ (=C-H stretching in furan ring) and at $3284 \mathrm{~cm}^{-1}(\mathrm{~N}-\mathrm{H}$ stretching vibration in pyrrole ring) in FTIR spectra. In ${ }^{1} \mathrm{H}-\mathrm{NMR}$, the signals of olefinic $\beta$-H and $\alpha-\mathrm{H}$ on double bond conjugated to the carbonyl group were seen at 7.68 and $7.46 \mathrm{ppm}$ as the both doublet $(\mathrm{J} \approx 15.02 \mathrm{~Hz})$ for $\mathbf{1 j}$ and at 7.68 and $7.52 \mathrm{ppm}$ as the both doublet $(\mathrm{J} \approx 15.60 \mathrm{~Hz})$ for $\mathbf{1 k}$.

The disappearance of carbonyl absorptions between $1660-1640 \mathrm{~cm}^{-1}$ in FT-IR spectra of chalcones $^{21}$ and arising a new band between 3290-3360 $\mathrm{cm}^{-1}$ (NH stretching vibration) confirm the cyclization to 2-pyrazoline derivatives of the chalcones. ${ }^{1} \mathrm{H}-\mathrm{NMR}$ spectra of 2-pyrazolines derivatives show a signal due to NH proton between 8.09-6.92 ppm as depending on substituted aromatic group bonded to pyrazoline ring at 5-position. 
Vicinal coupling constants of $\mathrm{NH}$ proton with pyrazoline ring proton at 5-position were in the range of $1.60-2.54 \mathrm{~Hz} .{ }^{13} \mathrm{C}$ NMR spectra of two pyrazolines ( $\mathbf{2} \mathbf{b}$ and $\left.\mathbf{2 i}\right)$ have also been taken and they show characteristic signals for 2-pyrazoline ring carbons at about $151 \mathrm{ppm}(\mathrm{C}=\mathrm{N}$ carbon $)$, at about $41 \mathrm{ppm}\left(\mathrm{CH}_{2}\right.$ ring carbon $)$ and at about $60 \mathrm{ppm}(\mathrm{CH}$ ring carbon).

\section{Biological activity}

All the synthesized chalcones and pyrazolines, except $\mathbf{1 j}$ and $\mathbf{1 k}$, were screened in vitro for antibacterial activity against Staphylococcus aureus COWAN 1, Pseudomonas aeruginosa DMS 50071 SCOTTA, Proteus vulgaris FMC 1, Escherichia coli ATCC 25922, Klebsiella pneumoniae FMC, Bacillus megaterium DMS 32 at absorbed $1000 \mu \mathrm{g}$ per disc and for antifungal activity against Candida albicans FMC 17, Candida glabrata ATCC 66032 and Candida tropicalis ATCC 13803 at $1000 \mu \mathrm{g}$ per disc by agar diffusion method ${ }^{22}$ given in detail in experimental section .

Antibacterial and antifungal activities of the obtained compounds were measured as a function of diameter of zone of inhibition $(\mathrm{mm})$. DMSO used as control showed activity at different ratios against the strains of all the bacteria and yeast. The results were compared with standard drugs amoksilin for antibacterial activitiy and nystatin for antifungal activity by measuring the zone of inhibition in $\mathrm{mm}$ at $1000 \mu \mathrm{g}$. Compound $\mathbf{2} \mathbf{j}$ was found most effective of the synthesized compounds against $S$. aureus with zone of inhibition $23 \mathrm{~mm}$, compound 1a is the most effective against $P$. aeruginosa and $P$. vulgaris with zone of inhibition $16 \mathrm{~mm}, \mathbf{2 k}$ is the most effective against $K$. pneumoniae and E. coli with zone of inhibition $18 \mathrm{~mm}$ and $17 \mathrm{~mm}$, respectively, and compound $1 \mathbf{a}$ is the most effective among the synthesized compounds against $B$. megaterium with zone of inhibition $27 \mathrm{~mm}$. The compound $\mathbf{2 j}$ exhibited the maximum diameter of zone of inhibition against $C$. albicans, C. glabrata and C. tropicalis $(21 \mathrm{~mm}, 23 \mathrm{~mm}$ and $20 \mathrm{~mm}$, respectively). Compounds $\mathbf{1 d}, \mathbf{1 f}, \mathbf{1 g}, \mathbf{1 h}, \mathbf{1 i}, \mathbf{2 d}$ and $\mathbf{2 h}$ showed no activity against the strains of all the bacteria and the yeast used in the work. Although compounds 1a, 2f, $2 \mathbf{g}$ and $2 \mathbf{i}$ showed an activity against most bacteria, they showed no activity against the yeast. The results of the antibacterial and antifungal activity are given in Table 1 .

Table 1. Antimicrobial and antifungal studies of synthesized compounds

\begin{tabular}{|c|c|c|c|c|c|c|c|c|c|c|c|c|c|c|c|}
\hline \multirow{3}{*}{ Bacteria/Yeast } & \multicolumn{15}{|c|}{ Zone of inhibition/ mm } \\
\hline & \multicolumn{15}{|c|}{ Compound numbers } \\
\hline & $1 \mathbf{a}$ & $1 \mathrm{~b}$ & 1c & $1 \mathrm{e}$ & $2 \mathbf{a}$ & $2 \mathbf{b}$ & $2 c$ & $2 e$ & $2 f$ & $2 \mathrm{~g}$ & $2 \mathbf{i}$ & $2 \mathbf{j}$ & $2 \mathbf{k}$ & $\mathbf{S}^{*}$ & D \\
\hline S. aureus & 13 & 10 & - & - & 13 & 13 & 13 & 10 & 9 & 12 & 10 & 23 & 19 & 38 & 10 \\
\hline P. aeruginosa & 16 & 15 & 10 & 10 & 12 & 15 & 9 & 14 & 9 & 14 & 10 & 9 & 10 & 28 & 8 \\
\hline P. vulgaris & 16 & 15 & 11 & 9 & 13 & 13 & 11 & 9 & - & - & 9 & 10 & 14 & 30 & 8 \\
\hline E. coli & 12 & 10 & 10 & 11 & 15 & 10 & 13 & 10 & - & - & 13 & - & 17 & 34 & 8 \\
\hline K. pneumoniae & 15 & 11 & 9 & - & 10 & 11 & 13 & 9 & 9 & 9 & 10 & - & 18 & 25 & 9 \\
\hline B. megaterium & 27 & 13 & 10 & 10 & 13 & 15 & 12 & 10 & 9 & 11 & 11 & 20 & 19 & 30 & 9 \\
\hline C. albicans & - & 11 & 10 & 10 & 10 & 9 & 11 & 11 & - & - & - & 21 & 20 & 27 & 9 \\
\hline C. glabrata & - & $\overline{-}$ & 10 & 11 & 10 & 9 & 15 & 14 & - & - & - & 23 & 19 & 32 & 10 \\
\hline C. tropicalis & - & 18 & 10 & 10 & 10 & 9 & 11 & 11 & - & - & - & 20 & 18 & 32 & 9 \\
\hline
\end{tabular}

*Antiacterial Standard Drag: Amoksilin $1000 \mu \mathrm{g}$, Antifungal Standard Drag: Nystatin $1000 \mu \mathrm{g}$ D: DMSO 
Another striking result is to show more or less activity against all the bacteria and the yeast of compounds $\mathbf{2 a}, \mathbf{2 b}, \mathbf{2 c}, \mathbf{2 e}$ and $\mathbf{2 k}$ which are pyrazolines with hydroxyl group moiety on the phenyl ring of their first four and the last is a pyrazoline having pyrrole ring. Compound 1a possessing hydroxyl group on ortho position of phenyl ring has shown very good antibacterial activity against $B$. megaterium and the activity is comparable with that of the standard drug (amoksilin) (with 90\% inhibition of that of the standard). Compound 1a against other bacteria and compounds $\mathbf{1 b}, \mathbf{2 a}, \mathbf{2 b}, \mathbf{2 c}, \mathbf{2 i}$ and $2 \mathbf{k}$ against all the bacteria showed moderate activity with 30-63\% inhibition of that of the standard drug. While compound $\mathbf{2} \mathbf{j}$ and $\mathbf{2 k}$ showed high antifungal activity against Candida albicans, Candida glabrata and Candida tropicalis, with 56-78\% inhibition of that of the standard drug (nystatin), compounds $\mathbf{1 c}, \mathbf{1 e}, \mathbf{2 a}, \mathbf{2 b}, \mathbf{2 c}$ and $\mathbf{2 e}$ showed moderate activity against all of the three yeast, with $28 \%-47 \%$ inhibition of that of the standard. The results obtained for antibacterial and antifungal activities of 2-pyrazoline derivatives used in this study are comparable with the values given in literature for some 2-pyrazolines ${ }^{23}$.

\section{Spectral Analysis of Compounds}

\section{1-(Benzofuran-2-yl)-3-(furan-2-yl)-2-propenon (1j)}

Yield: 77\%; m.p.122-124 ${ }^{\circ} \mathrm{C}$. FTIR ( $\left.\mathrm{KBr}, \mathrm{cm}^{-1}\right)$ : 3000-3150 (=C-H stretching in furan), 1659 $(\mathrm{C}=\mathrm{O}), 1597,1563,743 ;{ }^{1} \mathrm{H}-\mathrm{NMR}\left(300 \mathrm{MHz}, \mathrm{DMSO}-\mathrm{d}_{6}\right), \mathrm{ppm}: 7.71(\mathrm{~d}, \mathrm{~J}=7.3 \mathrm{~Hz}, 1 \mathrm{H}$, b4-H), $7.68(\mathrm{~d}, \mathrm{~J}=14.9 \mathrm{~Hz}, 1 \mathrm{H}, \beta-\mathrm{H}), 7.62(\mathrm{~d}, \mathrm{~J}=1.8 \mathrm{~Hz}, 1 \mathrm{H}, \mathrm{f} 5-\mathrm{H}), 7.61$ (d, J= $8.5 \mathrm{~Hz}, 1 \mathrm{H}$, b7-H), 7.55 (s, 1H, b3-H), 7.47 (dt, J= 8.5 and $1.2 \mathrm{~Hz}, 1 \mathrm{H}, \mathrm{b} 6-\mathrm{H}), 7.46(\mathrm{~d}, \mathrm{~J}=15.1 \mathrm{~Hz}, 1 \mathrm{H}$, $\alpha-\mathrm{H}), 7.31(\mathrm{t}, \mathrm{J}=8.2 \mathrm{~Hz}, 1 \mathrm{H}, \mathrm{b}-5 \mathrm{H}), 6.76(\mathrm{~d}, \mathrm{~J}=3.5 \mathrm{~Hz}, 1 \mathrm{H}, \mathrm{f} 3-\mathrm{H}), 6.52(\mathrm{dd}, \mathrm{J}=3.4$ and $1.8 \mathrm{~Hz}, 1 \mathrm{H}, \mathrm{f} 4-\mathrm{H}) .{ }^{13} \mathrm{C}-\mathrm{NMR}, \mathrm{ppm}: 178.6(\mathrm{C}=\mathrm{O}), 155.7$ (b8-C), 153.6 (b2-C), 151.4 (f2-C), 147.0 (f5-C), 130.4 ( $\beta-\mathrm{C}), 129.0$ (b6-C), 127.5 (b5-C), 124.6 (b4-C), $124.2(\alpha-\mathrm{C}), 121.1$ (b9-C), 118.9 (b3-C), 118.3 (f3-C), 114.8 (b7-C), 112.7 (f4-C). Anal. Calcd. For $\mathrm{C}_{15} \mathrm{H}_{10} \mathrm{O}_{3}$ : C, 75.62; H, 4.23\% Found C, 75.32; H, 4.27\%.

\section{1-(Benzofuran-2-yl)-3-(pyrrol-2-yl)-2-propenon (1k)}

Yield: 50\%; m.p.196-198 ${ }^{\circ} \mathrm{C}$. FTIR (KBr, cm $\left.{ }^{-1}\right)$ : 3284 (N-H stretching), 1648 (C=O), 1579,

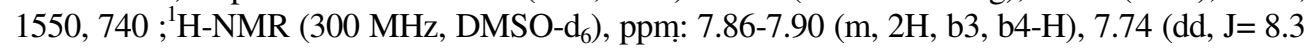
and $0.8 \mathrm{~Hz}, 1 \mathrm{H}, \mathrm{b} 7-\mathrm{H}), 7.68(\mathrm{~d}, \mathrm{~J}=15.7 \mathrm{~Hz}, 1 \mathrm{H}, \beta-\mathrm{H}), 7.55(\mathrm{dt}, \mathrm{J}=7.8$ and $1.2 \mathrm{~Hz}, 1 \mathrm{H}, \mathrm{b} 6-\mathrm{H}), 7.5$ $(\mathrm{d}, \mathrm{J}=15.5 \mathrm{~Hz}, 1 \mathrm{H}, \alpha-\mathrm{H}), 7.4(\mathrm{dt}, \mathrm{J}=7.5$ and $0.8 \mathrm{~Hz}, 1 \mathrm{H}, \mathrm{b} 5-\mathrm{H}), 7.19$ (broad signal, 1H, pyrrole-5$\mathrm{H}$ ), 6.81 (dd, J= 1.8 and $1.5 \mathrm{~Hz}, 1 \mathrm{H}$, pyrrol-4-H), 6.27 (broad signal, $1 \mathrm{H}$, pyrrole-3-H). Anal. Calcd. For $\mathrm{C}_{15} \mathrm{H}_{11} \mathrm{NO}_{2}$ : C, 75.94; H, 4.67; N, 5.90\% Found C, 75.55; H, 4.70; N, 6.20\%.

\section{3-(Benzofuran-2-yl)-5-(2-dihydroxphenyl)-2-pyrazoline (2a)}

Yield: 74\%; m.p.210-212 ${ }^{\circ} \mathrm{C}$. FTIR $\left(\mathrm{KBr}, \mathrm{cm}^{-1}\right): 3437(\mathrm{O}-\mathrm{H}), 3335(\mathrm{~N}-\mathrm{H}), ;{ }^{1} \mathrm{H}-\mathrm{NMR}(300 \mathrm{MHz}$, DMSO-d ${ }_{6}$ ), ppm: $9.64(\mathrm{~s}, 1 \mathrm{H}, \mathrm{OH}), 7.81(\mathrm{~d}, \mathrm{~J}=2.0 \mathrm{~Hz}, 1 \mathrm{H}, \mathrm{NH}), 7.56-7.63(\mathrm{~m}, 2 \mathrm{H}, \mathrm{b} 4, \mathrm{~b} 7-\mathrm{H})$, $7.32(\mathrm{dt}, \mathrm{J}=7.7$ and $1.4 \mathrm{~Hz}, 1 \mathrm{H}, \mathrm{b} 6-\mathrm{H}), 7.25(\mathrm{~m}, 2 \mathrm{H}, \mathrm{b} 5,6-\mathrm{H}), 7.09(\mathrm{dt}, \mathrm{J}=7.7$ and $1.7 \mathrm{~Hz}, 1 \mathrm{H}$, 4-H), 7.02 (s, 1H, b3-H), 6.85 (dd, J= 8.0 and $1.0 \mathrm{~Hz}, 1 \mathrm{H}, 3-\mathrm{H}), 6.67$ (ddd, J= 8.0, 2.4 and $0.6 \mathrm{~Hz}$, $1 \mathrm{H}, 3-\mathrm{H}), 5.08$ (dt, J= 10.3 and $1.9 \mathrm{~Hz}, 1 \mathrm{H}, \mathrm{p} 5-\mathrm{H}), 3.45$ (dd, $\mathrm{J}=16.1$ and $10.8 \mathrm{~Hz}, 1 \mathrm{H}, \mathrm{p} 4-\mathrm{H}), 2.78$ (dd, J= 16.1 and $9.7 \mathrm{~Hz}, 1 \mathrm{H}, \mathrm{p} 4-\mathrm{H}) .{ }^{13} \mathrm{C}-\mathrm{NMR}, \mathrm{ppm}: 155.1$ (b8-C), 154.8 (2-C), 151.1 (p3-C), 140.6 (b2-C), 128.7 (6-C), 128.7 (1-C), 128.5 (b9-C), 126.9 (4-C), 125.3 (b6-C), 123.7 (b5-C), 121.6 (b4-C), 119.4 (5-C), 115.5 (3-C), 111.5 (b7-C), 105.4 (b3-C), 58.8 (p5-C), 39.5 (p4-C). Anal. Calcd. For $\mathrm{C}_{17} \mathrm{H}_{14} \mathrm{~N}_{2} \mathrm{O}_{2}$ : C, 73.37; H, 5.07; N, 10.07\% Found C, 73.75; H, 4.85; N, 9.88\%.

\section{3-(Benzofuran-2-yl)-5-(3-dihydroxphenyl)-2-pyrazoline (2b)}

Yield: 80\%; m.p.219-221 ${ }^{\circ} \mathrm{C}$. FTIR (KBr, cm $\left.{ }^{-1}\right): 3410(\mathrm{O}-\mathrm{H}), 3335$ (N-H); ${ }^{1} \mathrm{H}-\mathrm{NMR}$ (300 MHz, DMSO-d $)_{6}$, ppm: 9.41 (s, 1H, OH), 7.85 (d, J=2.0 Hz, 1H,NH), 7.57-7.64 (m, 2H, 
b4, b7-H), 7.35 (dt, J= 7.6 and $1.4 \mathrm{~Hz}, 1 \mathrm{H}, \mathrm{b} 6-\mathrm{H}), 7.25$ (dt, J= 7.5 and $1.1 \mathrm{~Hz}, 1 \mathrm{H}, \mathrm{b} 5-\mathrm{H}), 7.14$ $(\mathrm{t}, \mathrm{J}=7.9,1 \mathrm{H}, 5-\mathrm{H}), 7.02(\mathrm{~s}, 1 \mathrm{H}, \mathrm{b} 3-\mathrm{H}), 6.78-6.80(\mathrm{~m}, 2 \mathrm{H}, 2,5-\mathrm{H}), 6.67$ (ddd, $\mathrm{J}=8.0,2.4$ and 0.6 $\mathrm{Hz}, 1 \mathrm{H}, 6-\mathrm{H}), 4.83(\mathrm{dt}, \mathrm{J}=10.4$ and $2.2 \mathrm{~Hz}, 1 \mathrm{H}, \mathrm{p} 5-\mathrm{H}), 3.45(\mathrm{dd}, \mathrm{J}=16.1$ and $10.8 \mathrm{~Hz}, 1 \mathrm{H}, \mathrm{p} 4-\mathrm{H})$, 2.86 (dd, J= 16.2 and $10.0 \mathrm{~Hz}, 1 \mathrm{H}, \mathrm{p} 4-\mathrm{H}) .{ }^{13} \mathrm{C}-\mathrm{NMR}, \mathrm{ppm}: 157.1$ (3-C), 154.8 (b8-C), 151.0 (p3-C), 144.6 (b2-C), 140.1 (1-C), 129.9 (5-C), 128.7 (b9-C), 125.4 (b6-C), 123.7 (b5-C), 121.6 (b4-C), 117.6 (6-C), 114.7 (2-C), 113.6 (4-C), 111.5 (b7-C), 105.4 (b3-C), 64.0(p5-C), 40.8(p4-C). Anal. Calcd. For $\mathrm{C}_{17} \mathrm{H}_{14} \mathrm{~N}_{2} \mathrm{O}_{2}$ : C, 73.38; H, 5.04; N, 10.07\% Found C, 73.82; H, 5.15; N, 9.97\%.

\section{3-(Benzofuran-2-yl)-5-(4-dihydroxphenyl)-2-pyrazoline (2c)}

Yield: 76\%; m.p.182-184 ${ }^{\circ} \mathrm{C}$. FTIR (KBr, cm $\left.{ }^{-1}\right): 3430(\mathrm{O}-\mathrm{H}), 3358(\mathrm{~N}-\mathrm{H}), 1610,808,750$; ${ }^{1} \mathrm{H}-\mathrm{NMR}\left(400 \mathrm{MHz}, \mathrm{CDCl}_{3}\right.$ ), ppm: $7.56(\mathrm{~d}, 1 \mathrm{H}, \mathrm{J}=8.5 \mathrm{~Hz}, \mathrm{~b} 4-\mathrm{H}), 7.51(\mathrm{~d}, 1 \mathrm{H}, \mathrm{J}=7.2, \mathrm{~b} 7-\mathrm{H})$, $7.31(\mathrm{dt}, \mathrm{J}=8.2$ and $1.3 \mathrm{~Hz}, 1 \mathrm{H}, \mathrm{b} 6-\mathrm{H}), 7.20-7.24(\mathrm{~m}, 5 \mathrm{H}, \mathrm{b} 3, \mathrm{~b} 5, \mathrm{NH}, 2-\mathrm{H}), 6.78-6.84(\mathrm{~m}, 3 \mathrm{H}$, $\mathrm{OH}, 3,5-\mathrm{H}), 4.92(\mathrm{dt}, \mathrm{J}=9.6$ and $1.8 \mathrm{~Hz}, 1 \mathrm{H}, \mathrm{p} 5-\mathrm{H}), 3.45(\mathrm{dd}, \mathrm{J}=16.3$ and $10.7 \mathrm{~Hz}, 1 \mathrm{H}$, p4-H), 3.05 (dd, J= 16.3 and $9.9 \mathrm{~Hz}, 1 \mathrm{H}, \mathrm{p} 4-\mathrm{H})$. Anal. Calcd. For $\mathrm{C}_{17} \mathrm{H}_{14} \mathrm{~N}_{2} \mathrm{O}_{2}: \mathrm{C}, 73.38 ; \mathrm{H}$, 5.04; N, 10.07\% Found C, 73.25; H, 5.12; N, 9.78\%.

\section{3-(Benzofuran-2-yl)-5-(4-N,N-dimethylaminophenyl)-2-pyrazoline (2d)}

Yield: 78\%; m.p.161-163 ${ }^{\circ} \mathrm{C}$. FTIR (KBr, $\left.\mathrm{cm}^{-1}\right)$ :3343 (N-H), 1611, 829; ${ }^{1} \mathrm{H}-\mathrm{NMR}$ (300 MHz, DMSO-d $\mathrm{d}_{6}$, ppm: $7.83(\mathrm{~d}, \mathrm{~J}=2.3 \mathrm{~Hz}, 1 \mathrm{H}, \mathrm{NH}), 7.63(\mathrm{~d}, \mathrm{~J}=7.5 \mathrm{~Hz}, 1 \mathrm{H}, \mathrm{b} 4-\mathrm{H})$, $7.57(\mathrm{~d}, \mathrm{~J}=7.8 \mathrm{~Hz}, 1 \mathrm{H}, \mathrm{b} 7-\mathrm{H}), 7.32(\mathrm{dt}, \mathrm{J}=7.8$ and $1.5 \mathrm{~Hz}, 1 \mathrm{H}, \mathrm{b} 6-\mathrm{H}), 7.25$ (dt, $\mathrm{J}=7.5$ and $1.1 \mathrm{~Hz}, 1 \mathrm{H}, \mathrm{b} 5-\mathrm{H}), 7.18(\mathrm{~d}, \mathrm{~J}=8.7,2 \mathrm{H}, 2,6-\mathrm{H}), 7.01(\mathrm{~s}, 1 \mathrm{H}, \mathrm{b} 3-\mathrm{H}), 6.70(\mathrm{~d}, \mathrm{~J}=8.8 \mathrm{~Hz}, 2 \mathrm{H}$, 3,5-H), 4.81 (dt, $\mathrm{J}=10.5$ and $2.3 \mathrm{~Hz}, 1 \mathrm{H}, \mathrm{p} 5-\mathrm{H}), 3.38(\mathrm{dt}, \mathrm{J}=16.1$ and $10.7 \mathrm{~Hz}, 1 \mathrm{H}, \mathrm{p} 4-\mathrm{H})$, 2.85 (dd, J= 16.3 and $10.5 \mathrm{~Hz}, 1 \mathrm{H}, \mathrm{p} 4-\mathrm{H})$. Anal. Calcd. For $\mathrm{C}_{19} \mathrm{H}_{19} \mathrm{~N}_{3} \mathrm{O}: \mathrm{C}, 74.73 ; \mathrm{H}, 6.27$; $\mathrm{N}, 13.76 \%$ Found C, 75.05; H, 6.05; N, 13.90\%.

\section{3-(Benzofuran-2-yl)-5-(3-methoxy-4-dihydroxphenyl)-2-pyrazoline (2e)}

Yield: 76\%; m.p.123-125 ${ }^{\circ} \mathrm{C}$. FTIR (KBr, cm $\left.{ }^{-1}\right): 3526(\mathrm{OH}), 3227(\mathrm{NH}), 1595,1524,1258$ (C-O-C), $751 ;{ }^{1} \mathrm{H}-\mathrm{NMR}\left(400 \mathrm{MHz}, \mathrm{CDCl}_{3}\right.$ ), ppm : 7.56 (d, 1H, J= 7.5 Hz, b4-H), 7.51 $(\mathrm{d}, 1 \mathrm{H}, \mathrm{J}=7.6, \mathrm{~b} 7-\mathrm{H}), 7.40(\mathrm{~s}, 1 \mathrm{H}, \mathrm{b} 3-\mathrm{H}), 7.30(\mathrm{t}, \mathrm{J}=7.7 \mathrm{~Hz}, 1 \mathrm{H}, \mathrm{b} 6-\mathrm{H}), 7.22(\mathrm{t}, \mathrm{J}=7.5 \mathrm{~Hz}$, $1 \mathrm{H}, \mathrm{b} 5-\mathrm{H}), 6.92(\mathrm{~d}, \mathrm{~J}=1.6 \mathrm{~Hz}, 1 \mathrm{H}, \mathrm{NH}), 6.82-6.88(\mathrm{~m}, 4 \mathrm{H}, \mathrm{OH}, 2,3,6-\mathrm{H}), 4.92(\mathrm{dt}, \mathrm{J}=9.4$ and $1.4 \mathrm{~Hz}, 1 \mathrm{H}, \mathrm{p} 5-\mathrm{H}), 3.47$ (dd, J= 16.3 and $10.8 \mathrm{~Hz}, 1 \mathrm{H}, \mathrm{p} 4-\mathrm{H}), 3.06$ (dd, $\mathrm{J}=16.2$ and $9.5 \mathrm{~Hz}$, 1H, p4-H). Anal. Calcd. For $\mathrm{C}_{18} \mathrm{H}_{16} \mathrm{~N}_{2} \mathrm{O}_{3}: \mathrm{C}, 70.12 ; \mathrm{H}, 5.23 ; \mathrm{N}, 9.09 \%$ Found $\mathrm{C}, 70.35 ; \mathrm{H}$, $5.08 ; \mathrm{N}, 9.27 \%$.

\section{3-(Benzofuran-2-yl)-5-(3-nitrophenyl)-2-pyrazoline (2f)}

Yield: 60\%; m.p.125-127 ${ }^{\circ} \mathrm{C}$. FTIR $\left(\mathrm{KBr}, \mathrm{cm}^{-1}\right)$ :3348 (N-H), $1523\left(\mathrm{NO}_{2}\right), 1350\left(\mathrm{NO}_{2}\right)$, ${ }^{1} \mathrm{H}-\mathrm{NMR}\left(400 \mathrm{MHz}, \mathrm{CDCl}_{3}\right)$, ppm : $8.35(\mathrm{~s}, 1 \mathrm{H}, 2-\mathrm{H}), 8.15(\mathrm{~d}, \mathrm{~J}=8.6 \mathrm{~Hz}, 1 \mathrm{H}, 4-\mathrm{H})$, 7.60-7.75 (m, 2H, 5,6-H), 7.45-7.55 (m, 2H, b4,b7-H), 7.20-7.35 (m, 3H, b5,b6-H,NH), 7.05 (s, 1H, b3-H), $5.20(\mathrm{t}, \mathrm{J}=10.1 \mathrm{~Hz}, 1 \mathrm{H}, \mathrm{p} 5-\mathrm{H}), 3.52(\mathrm{dd}, \mathrm{J}=16.8$ and $10.0 \mathrm{~Hz}, 1 \mathrm{H}, \mathrm{p} 4-\mathrm{H}), 3.05$ (dd, J=16.8 and $10.0 \mathrm{~Hz}, 1 \mathrm{H}, \mathrm{p} 4-\mathrm{H}$ ). Anal. Calcd. For $\mathrm{C}_{17} \mathrm{H}_{13} \mathrm{~N}_{3} \mathrm{O}_{3}: \mathrm{C}, 66.44 ; \mathrm{H}, 4.26 ; \mathrm{N}$, $13.67 \%$ Found C, 67.01; H, 4.15; N, 14.20\%.

\section{3-(Benzofuran-2-yl)-5-phenyl-2-pyrazoline (2g)}

Yield: 81\%; m.p.113-115 ${ }^{\circ} \mathrm{C}$, FTIR (KBr, cm $\left.{ }^{-1}\right): 3347(\mathrm{~N}-\mathrm{H}), 3100-3000,1610,1587,747$, 698; ${ }^{1} \mathrm{H}-\mathrm{NMR}$ (300 MHz, DMSO-d ${ }_{6}$ ), ppm: 8.01 (d, J=2.6 Hz, 1H, p-NH), 7.58-7.65 (m, 2H, b4,b7-H), 7.22-7.40 (m, 7H, 2-6,b5,b6-H), 7.02 (s, 1H, b3-H), 4.92 (dt, J=10.7 and $2.6 \mathrm{~Hz}, 1 \mathrm{H}, \mathrm{p} 5-\mathrm{H}), 3.48$ (dd, J= 16.1 and $10.4 \mathrm{~Hz}, 1 \mathrm{H}, \mathrm{p} 4-\mathrm{H}), 2.90$ (dd, $\mathrm{J}=16.1$ and $10.9 \mathrm{~Hz}$, 1H, p4-H). Anal. Calcd. For $\mathrm{C}_{17} \mathrm{H}_{14} \mathrm{~N}_{2} \mathrm{O}$ : C, 77.84; H, 5.38; N, 10.68\% Found C, 78.22; H, $5.25 ; \mathrm{N}, 10.98 \%$. 


\section{1,4-Bis[3-(benzofuran-2-yl)-2-pyrazoline-5-yl] benzene (2h).}

Yield: $45 \%$; m.p. $254-256{ }^{\circ} \mathrm{C}$. FTIR (KBr, $\left.\mathrm{cm}^{-1}\right)$ : ${ }^{1} \mathrm{H}-\mathrm{NMR}$ (300 MHz,DMSO-d $\mathrm{d}_{6}$ ), ppm: 7.99 (broad, 2H, p-NH), 7.62 (d, J= 7.2 Hz, 2H, b4-H), 7.58 (d, J= 7.8 Hz, 2H, b7-H), 7.38 (s, 4H, 2,3,5,6-H), 7.32 (t, J= 8.6 Hz,1H, b6-H), 7.29 (t, J= 7.5 Hz,1H, b5-H), 7,02 (s, 2H, b3-H), 4.92 (broad, t, J=10.2 Hz, 2H, p5-H), 3.47 (dd, J=16.2 and $10.9 \mathrm{~Hz}, 2 \mathrm{H}, \mathrm{p} 4-\mathrm{H}), 2.89$ (dd, J= 16.1 and $10.4 \mathrm{~Hz}, 2 \mathrm{H}, \mathrm{p} 4-\mathrm{H}$ ). Anal. Calcd. For $\mathrm{C}_{28} \mathrm{H}_{22} \mathrm{~N}_{4} \mathrm{O}_{2}: \mathrm{C}, 75.34 ; \mathrm{H}, 4.93 ; \mathrm{N}$, $12.56 \%$ Found C, 76.12; H, 5.20; N, 11.96\%.

\section{3-(Benzofuran-2-yl)-5-(thiophene-2-yl)-2-pyrazoline (2i)}

Yield: 90\%; m.p.114-116 ${ }^{\circ} \mathrm{C}$. FTIR $\left(\mathrm{KBr}, \mathrm{cm}^{-1}\right)$ : $3296(\mathrm{~N}-\mathrm{H}), 1601,808,750,700 ;{ }^{1} \mathrm{H}-\mathrm{NMR}$ (300 MHz, DMSO-d d , ppm: 8.09 (d, J=2.5 Hz, 1H, NH), 7.58-7.66 (m, 2H, b4,b7-H), 7.44 $(\mathrm{dd}, \mathrm{J}=5.0$ and $1.1 \mathrm{~Hz}, 1 \mathrm{H}, \mathrm{t} 3-\mathrm{H}), 7.34(\mathrm{dt}, \mathrm{J}=7.5 \mathrm{~Hz}$ and $1.0 \mathrm{~Hz}, 1 \mathrm{H}, \mathrm{b} 5-\mathrm{H}), 7.26$ (dt, $\mathrm{J}=7.3 \mathrm{~Hz}$ and $1.4 \mathrm{~Hz}, 1 \mathrm{H}, \mathrm{b} 6-\mathrm{H}), 7.08-7.10(\mathrm{~m}, 2 \mathrm{H}, \mathrm{t} 5, \mathrm{~b} 3-\mathrm{H}), 7.00(\mathrm{dd}, \mathrm{J}=5.1$ and $3.47 \mathrm{~Hz}$, $1 \mathrm{H}, \mathrm{t} 4-\mathrm{H}), 5.18(\mathrm{dt}, \mathrm{J}=10.3$ and $2.5 \mathrm{~Hz}, 1 \mathrm{H}, \mathrm{p} 5-\mathrm{H}), 3.49$ (dd, $\mathrm{J}=16.1$ and $10.6 \mathrm{~Hz}$ ), 2.95 (dd, $\mathrm{J}=16.1$ and $10.2 \mathrm{~Hz}) .{ }^{13} \mathrm{C}-\mathrm{NMR}$, ppm : 154.9 (b8-C), 150.6 (p3-C), 146.4 (b2-C), 141.0 (t2-C), 128.6 (b9-C), 127.3 (t4-C), 125.5 (t3-C), 125.4 (b6-C), 125.3 (t5-C), 123.7 (b5-C), 121.7 (b4C), 111.6 (b7-C), 105.9 (b3-C), 59.8 (p5-C), 41.5 (p4-C). Anal. Calcd. For $\mathrm{C}_{15} \mathrm{H}_{12} \mathrm{~N}_{2} \mathrm{OS}$ : C, 67.16; H, 4.48; N, 10.45; S, 11.94\% Found C, 67.23; H, 4.32; N, 10.63; S, $11.76 \%$.

\section{3-(Benzofuran-2-yl)-5-(furan-2-yl)-2-pyrazoline (2j)}

Yield: 77\%; m.p.92-94 ${ }^{\circ}$ C. FTIR (KBr, $\left.\mathrm{cm}^{-1}\right)$ : 3318, 3150-3000, 1605, 1258, 1154, 747; ${ }^{1} \mathrm{H}-\mathrm{NMR}\left(400 \mathrm{MHz}, \mathrm{CDCl}_{3}\right)$, ppm: $7.91(\mathrm{~d}, \mathrm{~J}=2.1 \mathrm{~Hz}, 1 \mathrm{H}, \mathrm{NH}), 7.57-7.67$ (m, 3H, b4,b7, f5-H), $7.33(\mathrm{dt}, \mathrm{J}=8.3$ and $1.4 \mathrm{~Hz}, 1 \mathrm{H}, \mathrm{b} 5-\mathrm{H}), 7.26(\mathrm{dt}, \mathrm{J}=7.3$ and $1.1 \mathrm{~Hz}, 1 \mathrm{H}, \mathrm{b} 6-\mathrm{H}), 7.07$ (s, 1H, b3-H), 6.38-6.43(m, 2H, f3,f4-H), 4.95 (dt, J= 10.1 and $2.1 \mathrm{~Hz}, 1 \mathrm{H}, \mathrm{p} 5-\mathrm{H}), 3.37$ (dd, $\mathrm{J}=15.6$ and $11.5 \mathrm{~Hz}, 1 \mathrm{H}, \mathrm{p} 4-\mathrm{H}), 3.14(\mathrm{dd}, \mathrm{J}=16.2$ and $9.2 \mathrm{~Hz}, \mathrm{p} 4-\mathrm{H})$. Anal. Calcd. For $\mathrm{C}_{15} \mathrm{H}_{12} \mathrm{~N}_{2} \mathrm{O}_{2}$ : C, 71.43; H, 4.76; N, 11.11\% Found C, 71.27; H, 4.55; N, 11.04\%.

\section{3-(Benzofuran-2-yl)-5-(pyrrol-2-yl)-2-pyrazoline (2k)}

Yield: 93\%; m.p.175-177 ${ }^{\circ} \mathrm{C}$. FTIR $\left(\mathrm{KBr}, \mathrm{cm}^{-1}\right): 3312,3190,3130,1610,1541 ;{ }^{1} \mathrm{H}-\mathrm{NMR}$ (400 MHz, $\mathrm{CDCl}_{3}$ ), ppm: 11.81 (broad, $1 \mathrm{H}, \mathrm{NH}$ ), 8.50 (broad, 1H, pyrrole-NH), 7.56 $(\mathrm{d}, \mathrm{J}=8.7 \mathrm{~Hz}, 1 \mathrm{H}, \mathrm{b} 4-\mathrm{H}), 7.53(\mathrm{~d}, \mathrm{~J}=8.6 \mathrm{~Hz}, 1 \mathrm{H}, \mathrm{b} 7-\mathrm{H}), 7.32(\mathrm{t}, \mathrm{J}=8.5 \mathrm{~Hz}, 1 \mathrm{H}, \mathrm{b} 6-\mathrm{H}), 7.24$ $(\mathrm{t}, \mathrm{J}=8.6 \mathrm{~Hz}, 1 \mathrm{H}, \mathrm{b} 5-\mathrm{H}), 6.82(\mathrm{~s}, 1 \mathrm{H}, \mathrm{b} 3-\mathrm{H}), 6.76(\mathrm{~m}, 1 \mathrm{H}$, pyrrole-5-H), 6.13-6.17 (m, 3H, p-NH, pyrrole-3,4-H), 5.05 (t, J=10.2 Hz, 1H, p5-H), $3.41(\mathrm{dd}, \mathrm{J}=16.3$ and $10.3 \mathrm{~Hz}, 1 \mathrm{H}$, p4-H), 3.09 (dd, J= 16.30and 10.1 Hz, 1H, p4-H). Anal. Calcd. For $\mathrm{C}_{15} \mathrm{H}_{13} \mathrm{~N}_{3} \mathrm{O}: \mathrm{C}, 71.71 ; \mathrm{H}$, 5.18; N, 16.73\% Found C, 71.52; H, 5.20; N, 17.02\%.

\section{Conclusion}

Syntheses and structural characterizations of two new chalcones and eleven pyrazolines were given in this study. The cyclization of the chalcones, given 2-pyrazoline was carried out at a shorter period of time than that of the some other chalcones given in literature using absolute ethanol in reflux. Antibacterial and antifungal activities of twenty compounds from this new class of benzofurane chalcones and pyrazoline derivatives against the six bacteria and three yeast were investigated. Seven compounds (1d, $\mathbf{1 f}, \mathbf{1 g}, \mathbf{1 h}, \mathbf{1 i}, \mathbf{2 d}$ and $\mathbf{2 h}$ ) showed no activity against any bacteria and any yeast. Five compounds $(\mathbf{2 a}, \mathbf{2 b}, \mathbf{2 c}, \mathbf{2 e}$ and $\mathbf{2 k})$ which are the all pyrazoline derivatives have exhibited an activity against all of the bacteria and yeast. Compound 1a possessing hydroxyl group on ortho position of phenyl ring has exhibited a comparable antibacterial activity against $B$. megaterium with that of the standard drug (amoksilin). While the compounds $\mathbf{1 b}, \mathbf{1 c}, \mathbf{1 e}$ and $\mathbf{2} \mathbf{j}$ are effective against most of bacteria and yeast used in this work, the compounds $2 \mathbf{f}, \mathbf{2 g}$ and $\mathbf{2 i}$ are effective against most bacteria but no yeast. 


\section{Acknowledgment}

The authors thank Firat University (FUBAP-1305) for financial support of this project.

\section{References}

1. Bhat B A, Dhar K L, Puri S C, Saxena A K, Shanmugavel M and Qazi G N, Bioorg Med Chem Lett., 2005, 15, 3177-3180.

2. Ansari F L, Umbreen S, Hussain L, Makhmoor T, Nawaz S A, Lodhi M A, Khan S N, Shaheen F, Choudhary M I and Rahman A, Chem Biodivers., 2005, 2(4), 487-496.

3. Jurasek A, Knoppava V, Dandarova M, Kovac J and Reinprecht L, Tetrahedron, 1978, 34, 1833-1836.

4. Trivedi J C, Bariwal J B, Upadhyay K D, Naliapara Y T, Joshi S K, Pannecouque C C, Clercq E D and Shah A K, Tetrahedron Lett., 2007, 48, 8472-8474.

5. Mahmood N, Piacente S, Burke A, Khan A and Pizza C, Antiviral Chem Chemother., 1997, 8, 70-74.

6. Domínguez J N, León C, Rodrigues J, de Domínguez N G, Gut J, Philip J and Rosenthal P J, Farmaco, 2005, 60, 307-311.

7. Li R, Kenyon G L, Cohen F E, Chen X, Gong B, Dominguez, J N, Davidson E, Kurzban G, Miller R E, Nuzum E O, Rosenthal P J and McKerrow J H, J Med Chem., 1995, 38(26), 5031-5037.

8. Chen M, Theander T G, Christensen S B, Hviid L, Zhai L and Kharazmi A, Antimicrob Agents Chemother, 1994, 38, 1470-1475.

9. Khatib S, Narya O, Musa R, Shmuel M, Tamir S and Vaya J, Bioorg Med Chem., 2005, 13, 433-441.

10. Devia C M, Pappano N B and Debattista N B, Rev Microbiol., 1998, 29(4), 307-310.

11. Yaulì $\mathrm{N}$, Üçüncü $\mathrm{O}$, Aydìn $\mathrm{E}$, Gök $\mathrm{Y}$, Yasar $\mathrm{A}$, Baltacì Yìldìrìm $\mathrm{C} \mathrm{N}$, Küçük $\mathrm{M}, J$ Photochem Photobiol A: Chem., 2005, 169, 229-234.

12. Williams C A, Hoult J R S, Harborne J B, Greenham J and Eagles J, Phytochem., 1995, 38, 267-270.

13. Nauduri D and Reddy G B, Chem Pharm Bull Tokyo, 1998, 46, 1254-1260.

14. Babu V H, Sridevi C H, Joseph A and Srinivasan K K, Ind J Pharm Sci., 2007, 69(3), 470-473.

15. Korgaokar S S, Patil P H, Shah M T and Parekh H H, Indian J Pharm Sci., 1996, 58, 222-224.

16. Udupi R H, Kushnoor A S and Bhat A R, Indian J Heterocycl Chem., 1998, 8(1), 63-66.

17. Jayamma Y, Sarangapani M and Reddy V M, Indian J Heterocycl Chem., 1996, 6, 111-114.

18. Johnson M, Younglove B, Lee L, LeBlanc R, Holt H Jr, Hills P, Mackay H, Brown T, Mooberry S L and Lee M, Biorg Med Chem Lett., 2007, 17(21), 5897-5901.

19. Bilgin A A, Palaska E and Sunal R, Arzneimforsch, Drug Res., 1993, 43, 1041-1044.

20. Manna F, Chimenti F, Fioravanti R, Bolasco A, Secci D, Chimenti P, Ferlini C and Scambia G, Bioorg Med Chem Lett., 2005, 15(20), 4632-4635.

21. Coşkun D and Ahmedzade M, Synth Comm., 2008, 38, 3613-3622.

22. Chan E C S, Pelczar M J and Krieg N R, Agar-diffusion Methods, In Laboratory Exercises In Microbiology, McGraw-Hill, New York, 1993, p225.

23. Kini S and Gandhi A M, Indian J Pharm Sci., 2008, 70, 105-108. 


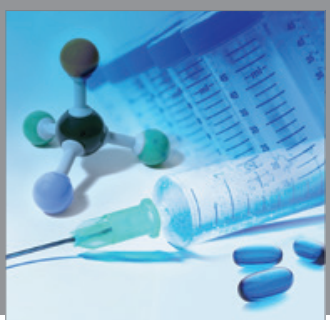

International Journal of

Medicinal Chemistry

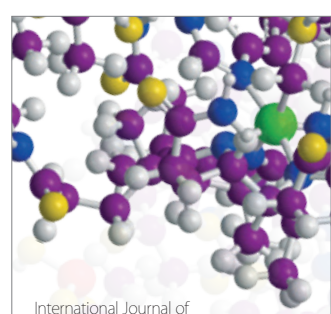

Carbohydrate Chemistry

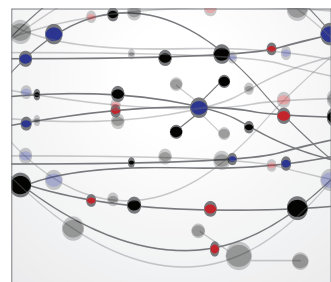

The Scientific World Journal
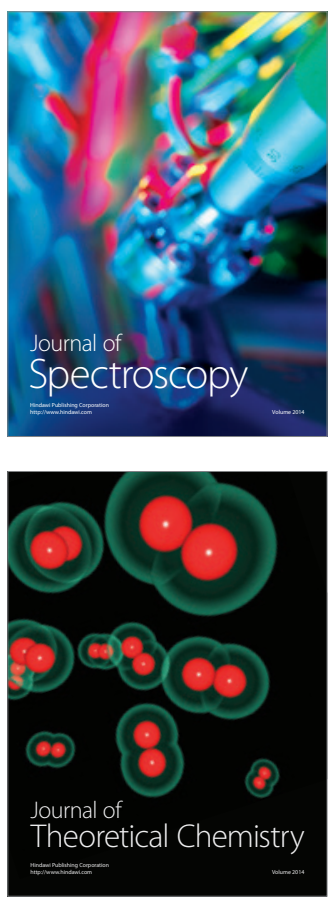
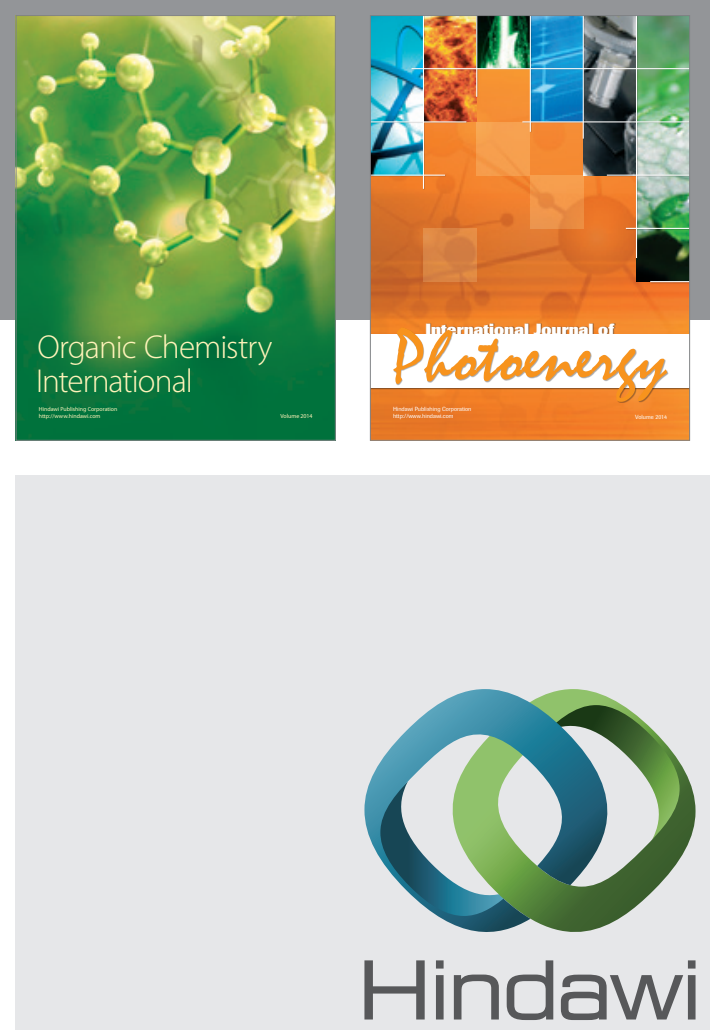

Submit your manuscripts at

http://www.hindawi.com
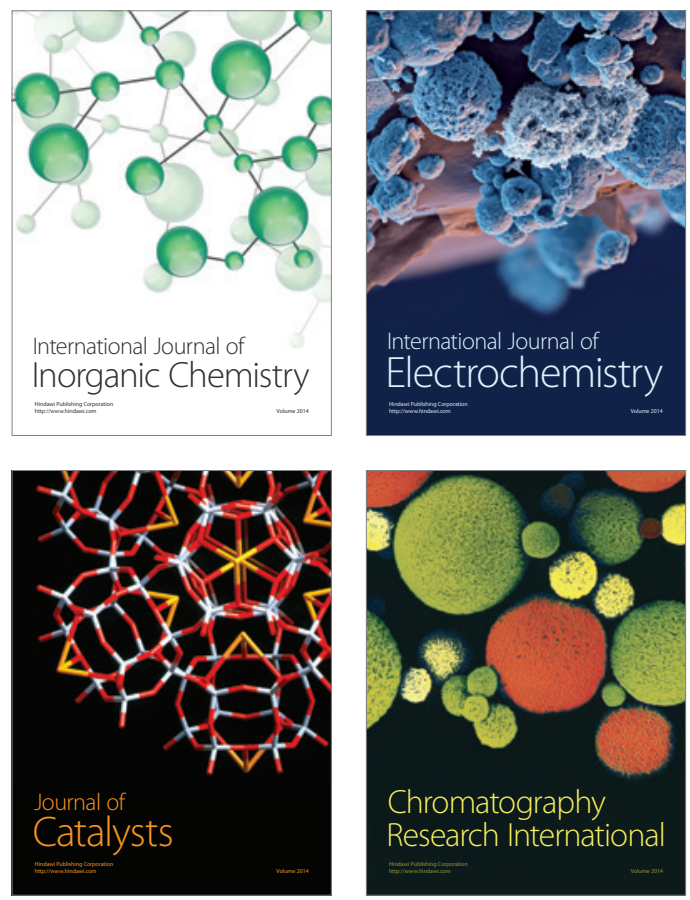
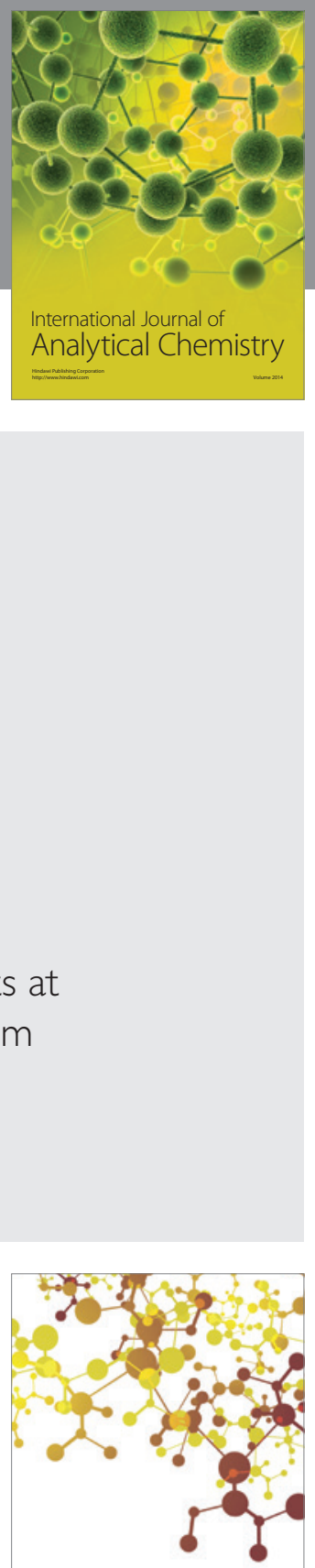

Journal of

Applied Chemistry
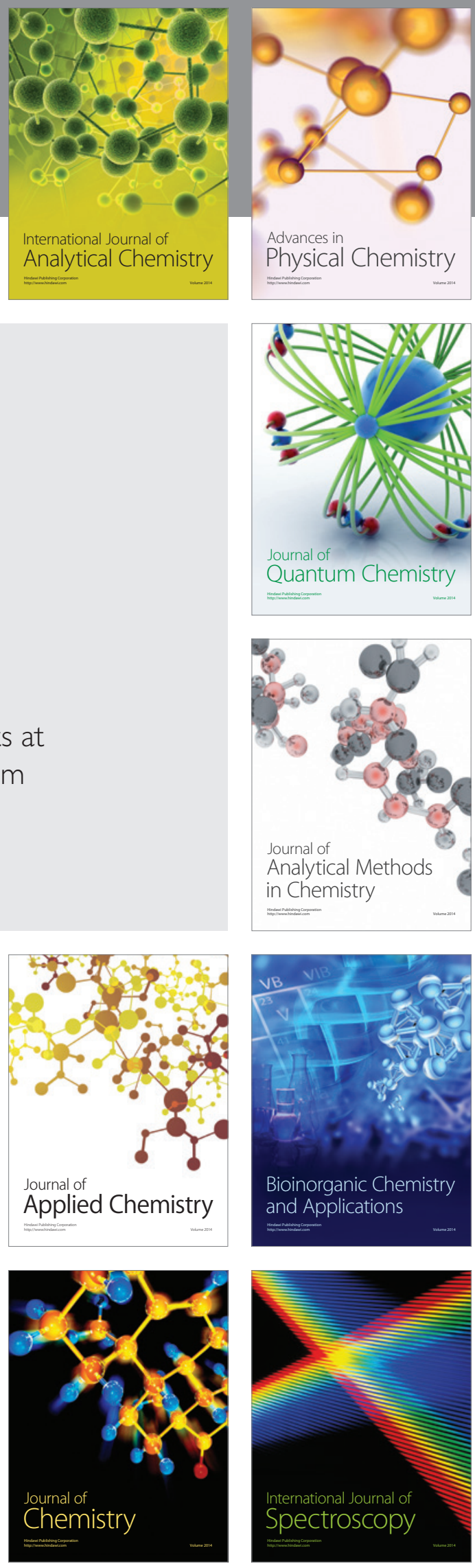